\title{
Robust Beamforming Algorithm Based on Linear Constrained Minimum Variance and Diagonal Loading
}

\author{
Chuan $\mathrm{HE}^{1}$, Yecai GUO ${ }^{2, \mathrm{a}^{*}}$, Xiaoyan $\mathrm{CHEN}^{2}$ and Lihua WANG ${ }^{2}$ \\ ${ }^{1}$ College of Atmospheric Physics, Nanjing University of Information Science and Technology, \\ Nanjing 210044, China \\ ${ }^{2}$ Jiangsu Collaborative Innovation Center on Atmospheric Environment and Equipment Technology \\ (CICAEET), Nanjing 210044,China \\ a* email: guo-yecai@163.com
}

Keyword: Robust microphone array beamforming; Linear constrainted minimum variance criterion; Diagonal loading; Spatial response variation function

\begin{abstract}
In order to improve the robustness of microphone array beamformer in existing mismatch errors, robust beamforming algorithm based on linear constrained minimum variance and diagonal loading method is proposed. The proposed algorithm incorporates the spatial response variation function into the linear constrainted minimum variance criterion to design the frequency invariant beamformer and improve the robustness against microphone array mismatch errors in the actual environment by diagonal loading method. Simulation results and analyses illustrate that the array response within passband is robust, the stopband amplitude is controlled in the appropriate range, and the proposed algorithm based closed solution is better than the proposed algorithm based on convex optimization algorithm.
\end{abstract}

\section{Introduction}

As one of the key technologies for microphone arrays, broadband beamforming has been used in a wide range of audio and speech processing applications[1]. In many popular methods, the traditional linear constrained minimum variance(LCMV) beamforming method is mainly used to design narrow-band antenna beamformer[2]. If it is used to design a broadband beamformer of microphone array, it isn't fit. It is necessary for microphone array to design broadband beamformer without specific arrays[3][4]. In order to achieve broadband frequency invariant beamformer[3], it is necessary to consider frequency invariant beamformer with mismatch errors caused by the gain, phase and position of the microphone[5][6]. For this purpose, in this paper, frequency invariant beamforming algorithm based on the linear constrainted minimum variance (LCMV), the spatial response variation function(SRV) of the microphone array, and diagonal loading method are used to design frequency invariant beamformer and improve the robustness on the given frequency range and the region.

\section{Array Model}

Consider a $M$-element linear microphone array in the far-field (not general, this method can be applied to arbitrary array structure), FIR subfilter with tap length $L$ is added to microphone. The array output signal can be expressed as $\boldsymbol{y}(k)=\boldsymbol{w}^{H} \boldsymbol{x}(k)$, where $H$ denotes conjugate transpose.The weight vector of microphone array response is written as $\boldsymbol{w}(k)=\left[w_{11}(k), \mathrm{L}, w_{M 1}(k), \mathrm{L}, w_{1 L}(k), \mathrm{L}, w_{M L}(k)\right]^{T} \quad, \quad T \quad$ denotes transpose. $\boldsymbol{x}(k)=\left[x_{11}(k), \mathrm{L}, x_{M 1}(k), \mathrm{L}, x_{1 L}(k), \mathrm{L}, x_{M L}(k)\right]^{T}$ and it denotes the received signal of microphone array, the definition of the angle between the direction of the sound source and the line array is $\theta$, the beam-pattern at frequency $f$ and angle $\theta$ of arrival can be expressed as $H(f, \theta)=\left|\boldsymbol{w}^{T} \boldsymbol{d}(f, \theta)\right|$, where frequency $f$ is belongs to $\left[f_{l}, f_{h}\right]$, the angle $\theta$ of the received signal is in $\left[\theta_{l}, \theta_{h}\right]$, and $\boldsymbol{d}(f, \theta)$ denotes the array response vector. 


\section{Frequency Invariant Beamforming Algorithm and Diagonal Loading Method}

The spatial response variation function(SRV) is given by

$$
S R V=\sum_{n=0}^{N-1} \sum_{q=0}^{Q-1}\left|\boldsymbol{w}^{H} \boldsymbol{d}\left(f_{n}, \theta_{q}\right)-\boldsymbol{w}^{H} \boldsymbol{d}\left(f_{r}, \theta_{q}\right)\right|^{2}=\boldsymbol{w}^{H} \boldsymbol{R}_{P S} \boldsymbol{w}=\boldsymbol{w}^{H}\left[(1-\beta) \boldsymbol{R}_{P}+\beta \boldsymbol{R}_{S}\right] \boldsymbol{w}
$$

where $f_{r}$ is reference frequency, $\boldsymbol{R}_{P S}$ is the equilibrium matrix of array spatial response variation function. $0<\beta<1$ is an equilibrium parameter bewteen frequency invariance and stopband attenuation. $\boldsymbol{R}_{P}$ is the matrix of the reference array response variation vector and $\boldsymbol{R}_{S}$ is reference array response matrix. The frequency invariant beamforming problem based on LCMV criterion and SRV can be expressed as

$$
\min _{\boldsymbol{w}} \boldsymbol{w}^{H}\left(\boldsymbol{R}_{x X}+\alpha \boldsymbol{R}_{P S}\right) \boldsymbol{w} \text { s.t. } \boldsymbol{C}^{H} \boldsymbol{w}=\boldsymbol{F}
$$

where $\quad \boldsymbol{R}_{x x}=E\left[x(k) x^{T}(k)\right], \quad \boldsymbol{C}=\left[\boldsymbol{d}\left(f_{0}, \theta\right), \quad \boldsymbol{d}\left(f_{1}, \theta\right), \mathrm{L}, \boldsymbol{d}\left(f_{n}, \theta\right), \quad \mathbf{L}, \boldsymbol{d}\left(f_{N-1}, \theta\right)\right]$, $\boldsymbol{F}=\left[e^{-j \pi f_{0}(L-1) f_{s}}, \mathrm{~L}, e^{-j \pi f_{1}(L-1) f_{s}}, \mathrm{~L}, e^{-j \pi f_{N-1}(L-1) f_{s}}\right]^{T}, \alpha$ is the matrix weighting factor and a positive number. When some uncertainties in microphone gain, phase, and position are considered[6], the microphone characteristics of the $m$-th microphone and the array response of the beamforming with the mismatch errors can be expressed as

$$
\mathscr{q}_{m}(f, \theta)=\left[1+g_{m}\right] e^{-j \varphi_{m}} e^{-j 2 \pi f \delta_{m} \cos \theta / c}, H / \varphi(f, \theta)=\mathscr{g} 0_{m}(f, \theta) H(f, \theta)
$$

where $g_{m}$ denotes gain error, $\delta_{m}$ denotes position error, and $\varphi_{m}$ denotes phase error. The diagonal loading LCMV frequency invariant beamforming problem is expressed as

$$
\min _{\boldsymbol{w}} \boldsymbol{w}^{H}\left(\boldsymbol{R}_{x x}+\alpha \boldsymbol{R}_{P S}\right) \boldsymbol{w} \text { s.t. } \boldsymbol{C}^{H} \boldsymbol{w}=\boldsymbol{F} \quad \boldsymbol{w}^{H} \boldsymbol{w} \leq \zeta
$$

where $\zeta$ is a constraint value and must meet $\zeta \geq 1 / M$. Using Lagrange method, the weight vector of the beamformer is given by

$$
\boldsymbol{w}_{\text {opt }}=\left(\boldsymbol{R}_{x x}+\alpha \boldsymbol{R}_{P S}+\lambda \boldsymbol{I}\right)^{-1} \boldsymbol{C}\left(\boldsymbol{C}^{H}\left(\boldsymbol{R}_{x x}+\alpha \boldsymbol{R}_{P S}+\lambda \boldsymbol{I}\right)^{-1} \boldsymbol{C}\right)^{-1} \boldsymbol{F}
$$

In order to overcome the defect of the diagonal loading method, according to the convex optimization toolbox,the problems in Eq.4 can be expressed as

$$
\min _{\boldsymbol{w}}\|\boldsymbol{L} \boldsymbol{w}\|^{2} \quad \text { s.t. }\left\|\boldsymbol{C}^{H} \boldsymbol{w}-\boldsymbol{F}\right\|^{2} \leq \zeta_{0} \quad\|\boldsymbol{w}\|^{2} \leq \zeta
$$

where $\boldsymbol{R}_{x x}+\alpha \boldsymbol{R}_{P S}=\boldsymbol{L}^{H} \boldsymbol{L}, \boldsymbol{w}^{H}\left(\boldsymbol{R}_{x x}+\alpha \boldsymbol{R}_{P S}\right) \boldsymbol{w}=\|\boldsymbol{L} \boldsymbol{w}\|^{2}, \quad \zeta_{0}$ is a desired signal constraint value. The diagonal loading can be obtained by

$$
\boldsymbol{w}^{H} \boldsymbol{w}=(\boldsymbol{C F})^{H}\left(\boldsymbol{R}_{x x}+\alpha \boldsymbol{R}_{P S}+\lambda \boldsymbol{I}\right)^{-2} \boldsymbol{C F}\left[\boldsymbol{C}^{H}\left(\boldsymbol{R}_{x x}+\alpha \boldsymbol{R}_{P S}+\lambda \boldsymbol{I}\right)^{-1} \boldsymbol{C}\right]^{-2}=\zeta
$$

where

$$
\begin{aligned}
& \zeta \leq \frac{\left(\gamma_{1}+\lambda\right)^{2}}{M L\left(\gamma_{M L}+\lambda\right)^{2}} \\
& \lambda \leq \frac{\gamma_{1}-(M L \zeta)^{1 / 2} \gamma_{M L}}{(M L \zeta)^{1 / 2}-1}
\end{aligned}
$$

The diagonal loading values in Eq.9 may be obtained by the Newton iteration method. $\gamma_{i}$ denotes the $i$ th eigenvalue of $\boldsymbol{R}, 1 \leq i \leq M L$. 


\section{Simulation Tests}

The effectiveness of the proposed algorithm was verified in existing microphone mismatch errors. An uniform linear array with 15 identical omnidirectional microphone was used. The order of FIR filter was 31, the array element spacing was the half wavelength of the highest frequency of $5 \mathrm{~cm}$, the sampling frequency was $8000 \mathrm{~Hz}$, the sound velocity was $340 \mathrm{~m} / \mathrm{s}$, the noise variance $\sigma_{n}^{2}=0.01$, the interference variance $\sigma_{i}^{2}=0.01$. The direction of the expected signal was $90^{\circ}$ and the limit of the gain error of the microphone was $g_{m}=0.2$, the phase error bound was $\varphi_{m}=\pi / 18$, the error bound for the $\mathrm{X}$ axis component of the position vector of the microphone was $\delta_{m}=0.02$.Simulation results was shown in Fig.1, (a) 3D array response of closed solution, (b) side view of array response of the closed solution, (c) 3D array response of the convex optimization solution, (d) side view of array response of the convex optimization.

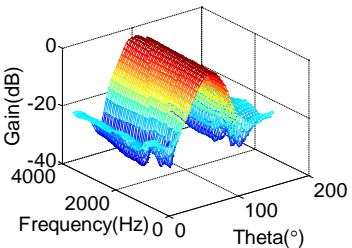

(a)

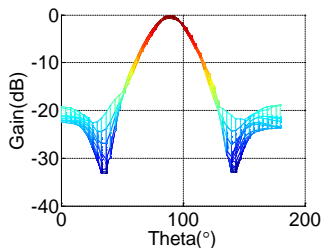

(b)

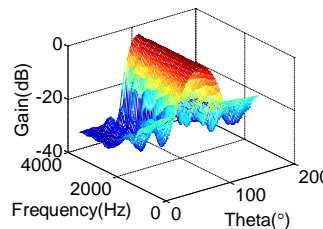

(c)

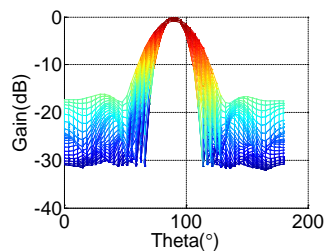

(d)

Figure 1 Array response of robust beamformer

From Fig.1, it can be seen that the array response within passband is robust, the stopband amplitude is controlled in the appropriate range (about $-20 \mathrm{~dB}$ or $-17 \mathrm{~dB}$ ), and the robust convex optimization algorithm has poor performance in the expected direction comparison with closed solution.

\section{Conclusion}

The robust linear constrained minimum variance beamforming algorithm against microphone mismatches has been proposed, which takes into consideration of diagonal loading method that imposes norm constraint to weight vector. In this proposed algorithm, the linear constrained minimum variance beamforming algorithm is combined with the SRV to obtain better frequency invariant property, the optimal weight vector is obtained by using Lagrange and convex optimization toolbox. respectively. Simulation results show that the proposed algorithm based closed solution is better than the proposed algorithm based on convex optimization algorithm.

\section{Acknowledgement}

This work is supported by the National Natural Science Foundation of China (51405241,61371131,11504176), the Major Project of Nature Science Foundation of Higher Education Institution of Jiangsu Province, China(Grant No.13KJA510001), Jiangsu Scientific Research Achievements in Industrialization Project, China(JHB 2012-9), and Jiangsu Province, the 2015 Annual General University Graduate Students Practice Innovation Program(SJLX15_0398).

\section{References}

[1] Benesty J, Chen J, Huang Y. Microphone array signal processing[M]. 2008, 164-166.

[2] H L Van Trees. Optimum Array Processing[M]. 2002, 76-78.

[3] RMM Derkx, K Janse. Theoretical analysis of a first-order azimuth-steerable superdirective microphone array[J]. IEEE Transaction on Audio, Speech, and Language Processing, 2009, 17(1): 150-162. 
[4] CC Lai, S Nordholm, YH Leung. Design of steerable spherical broadband beamformers with flexible sensor configuration[J]. IEEE Transaction on Audio, Speech, and Language Processing, 2013, 21(2): 427-438.

[5] W Liu, S Weiss. Wideband Beamforming: Concepts and Techniques[M]. 2010, 55-58.

[6] S Doclo, M Moonen. Design of far-field and near-field broadband beamformers using eigenfilters[J]. Signal Process, 2003, 83(12): 2641-2673.

[7] S Doclo, M Moonen. Design of broadband beamformers robust against gain and phase errors in the microphone array characteristics[J]. IEEE Trans. Signal Process, 2003, 51(10):2511-2526.

[8] H. Chen, W. Ser, and Z. L. Yu. Optimal design of nearfield wideband beamformers robust against errors in microphone array characteristics[J]. IEEE Transactions on Circuits Syst, 2007, 54(9): 1950-1959. 